PROCEEDINGS OF THE AMERICAN MATHEMATICAL SOCIETY

Volume 126, Number 3, March 1998, Pages 887-897

S $0002-9939(98) 04595-\mathrm{X}$

\title{
ON THE HOMOTOPY INVARIANCE OF $L^{2}$ TORSION FOR COVERING SPACES
}

\author{
VARGHESE MATHAI AND MELVIN ROTHENBERG
}

(Communicated by Jozef Dodziuk)

\begin{abstract}
We prove the homotopy invariance of $L^{2}$ torsion for covering spaces, whenever the covering transformation group is either residually finite or amenable. In the case when the covering transformation group is residually finite and when the $L^{2}$ cohomology of the covering space vanishes, the homotopy invariance was established by Lück. We also give some applications of our results.
\end{abstract}

\section{INTRODUCTION}

$L^{2}$ analytic torsion for $L^{2}$ acyclic covering spaces with positive decay was first studied in $[\mathrm{M}]$ and in $[\mathrm{L}]$, and $L^{2}$ Reidemeister-Franz torsion was first studied in $[\mathrm{CM}]$; see also $[\mathrm{LuR}],[\mathrm{Lu}]$. These $L^{2}$ torsion invariants were subsequently generalised by using the theory of determinant lines of finitely generated Hilbertian modules over finite von Neumann algebras, which was initiated in [CFM]. Non-zero elements of the determinant lines can be viewed as volume forms on the Hilbertian modules. Using this, the authors of $[\mathrm{CFM}]$ constructed both $L^{2}$ combinatorial torsion and $L^{2}$ analytic torsion invariants associated to flat Hilbertian bundles of determinant class over compact polyhedra and manifolds, as volume forms on the $L^{2}$ homology and $L^{2}$ cohomology, respectively, under the assumption that the covering space was of determinant class. These $L^{2}$ torsion invariants specialise to the RaySinger-Quillen torsion and the classical Reidemeister-Franz torsion, respectively, in the finite dimensional case. Using the results of [BFKM], it was shown in [CFM] that the combinatorial and analytic $L^{2}$ torsions were equal whenever the covering space is of determinant class. By this result and calculations done in $[\mathrm{M}]$ and $[\mathrm{L}]$, one obtains a simplicial description of hyperbolic volume for a closed 3-dimensional hyperbolic manifold in terms of the $L^{2}$ Reidemeister-Franz torsion.

In this paper, we prove the homotopy invariance of $L^{2}$ torsion for covering spaces, whenever the covering transformation group is either residually finite or amenable. In the case when the covering transformation group is residually finite and when the $L^{2}$ cohomology of the covering space vanishes, the homotopy invariance was established by Lück [Lu]. Using our main theorem, we can define the $L^{2}$ torsion of a discrete group $\Gamma$, where $\Gamma$ is either residually finite or amenable and such that

Received by the editors May 16, 1996.

1991 Mathematics Subject Classification. Primary 58G11, 58G18, 58G25.

Key words and phrases. $L^{2}$ torsion, invariants, amenable groups, residually finite groups, Whitehead groups, homotopy invariance.

The second author was supported in part by NSF Grant DMS 9423300. 
$B \Gamma$ is a finite $\mathrm{CW}$ complex. The $L^{2}$ torsion of the discrete group $\Gamma$ is then an element of the determinant line of the reduced $L^{2}$ cohomology of the group $\Gamma$. We also give a new proof of the classical result that the hyperbolic volume of a closed 3-dimensional hyperbolic manifold is a homotopy invariant, which actually only uses the part of our theorem which was proved by Lück [Lu]. Our paper relies on the recent results that residually finite covering spaces are of determinant class due to $[\mathrm{Lu}],[\mathrm{BFK}]$ and that amenable covering spaces are of determinant class due to [DM2]. The authors thank Shmuel Weinberger for some helpful conversations.

\section{Preliminaries}

1.1. Determinants and determinant lines. Here we will review some of the results on the Fuglede-Kadison determinant and on determinant lines of Hilbertian modules over finite von Neumann algebras. The results of this section are mainly from the paper $[\mathrm{CFM}]$. See also the papers $[\mathrm{CM}],[\mathrm{FK}]$, [Lu1] and $[\mathrm{LuR}]$.

Let $\mathcal{A}$ be a finite von Neumann algebra with a fixed faithful finite normal trace $\tau: \mathcal{A} \rightarrow \mathbb{C}$. Then $\mathcal{A}$ has a scalar product $\langle a, b\rangle=\tau\left(b^{*} a\right)$ for $a, b \in \mathcal{A}$, where ${ }^{*}$ denotes the involution on $\mathcal{A}$. Let $l^{2}(\mathcal{A})$ denote the completion of $\mathcal{A}$ with respect to this scalar product.

A Hilbert $\mathcal{A}$-module is a Hilbert space $M$ together with a continuous left $\mathcal{A}$ module structure such that there is an isometric $\mathcal{A}$-linear embedding of $M$ into $l^{2}(A) \otimes H$ for some Hilbert space $H(\mathcal{A}$ acts trivially on $H)$. Note that the embedding is not part of the structure. $M$ is said to be finitely generated if $H$ can be chosen to be finite dimensional.

A Hilbert module has a particular scalar product. If we ignore the scalar product, we get what we call a Hilbertian $\mathcal{A}$-module. More precisely a Hilbertian $\mathcal{A}$-module is a topological vector space $M$ with a left $\mathcal{A}$-module structure such that there is a scalar product $\langle$,$\rangle on M$ which generates the topology of $M$, and such that $(M,\langle\rangle$, is a Hilbert $\mathcal{A}$-module.

Any scalar product $\langle$,$\rangle on M$ as above will be called admissible. Suppose that $\langle,\rangle_{1}$ is another scalar product on $M$. Then there is an operator $A: M \rightarrow M$ such that $\langle v, w\rangle_{1}=\langle A v, w\rangle$, and which satisfies

(1) $A$ is a linear homeomorphism since $\langle$,$\rangle and \langle,\rangle_{1}$ define the same topology,

(2) $A$ is self adjoint with respect to $\langle$,$\rangle ,$

(3) $A$ is positive with respect to $\langle$,$\rangle ,$

(4) $A$ commutes with $\mathcal{A}$.

Any two admissible scalar products on a Hilbertian $\mathcal{A}$-module give rise to isomorphic Hilbert $\mathcal{A}$-modules. In particular, if we choose an admissible scalar product on $M$, it becomes a Hilbert $\mathcal{A}$-module, and the von Neumann dimension $\operatorname{dim}_{\tau}(M)$, which is defined as the von Neumann trace (defined in the next paragraph) of the orthogonal projection from $\ell^{2}(\mathcal{A}) \otimes H$ onto $M$, is independent of the choice of admissible scalar product.

The commutant of $M, B(M)$, is the algebra of all bounded linear operators commuting with $\mathcal{A}$. Then there is a canonical trace on $B(M), \operatorname{Tr}_{\tau}$, defined as follows: If $M$ is free and isomorphic to $l^{2}(\mathcal{A}) \otimes \mathbb{C}^{k}$, then $B(M)$ consists of $k \times k$ matrices with entries in $\mathcal{A}$, acting on the right, and for $f \in B(M)$,

$$
\operatorname{Tr}_{\tau}(f)=\sum_{i=1}^{k} \tau\left(f_{i i}\right)
$$


is the trace. In the general case, $\operatorname{Tr}_{\tau}(f)=\operatorname{Tr}_{\tau}\left(i_{M} \circ f \circ \pi_{M}\right)$, where $i_{M}$ denotes the embedding of $M$ into a free module and $\pi_{M}$ denotes the projection from the free module onto $M$.

Let $G L(M)$ denote the group of all invertible operators in the commutant of $M$, $B(M)$. Then there is a determinant function,

$$
\operatorname{Det}_{\tau}: G L(M) \rightarrow \mathbb{R}^{+},
$$

called the Fuglede-Kadison determinant, which satisfies

(1) $\operatorname{Det}_{\tau}(A B)=\operatorname{Det}_{\tau}(A) \operatorname{Det}_{\tau}(B)$,

(2) $\operatorname{Det}_{\tau}(\lambda I)=|\lambda|^{\operatorname{dim}_{\tau}(M)}$ for $\lambda \in \mathbb{C}$,

(3) $\operatorname{Det}_{\lambda \tau}(A)=\operatorname{Det}_{\tau}(A)^{\lambda}$ for $\lambda>0$,

(4) $\operatorname{Det}_{\tau}$ is continuous in the norm topology on $G L(M)$.

Its definition is as follows [CFM]: Let $A \in G L(M)$, and let $A_{t}$ be a piecewise smooth path from the identity to $A$ (since $G L(M)$ is connected, cf. [Di]). Define

$$
\log \operatorname{Det}_{\tau}(A)=\int_{0}^{1} \Re \operatorname{Tr}_{\tau}\left(A_{t}^{-1} \dot{A}_{t}\right) d t
$$

where $\Re$ denotes the real part. Using a result of Araki, Smith and Smith [ASS], if $t \rightarrow A_{t}$ is a loop, then $\int_{0}^{1} \Re \operatorname{Tr}_{\tau}\left(A_{t}^{-1} \dot{A}_{t}\right) d t=0$, so that (1.1) is well defined. The definition is extended to operators $A \in B(M)$ as follows: let $|A|=\int_{0}^{\infty} \lambda d E_{\lambda}$ denote the spectral decomposition of $|A|=\sqrt{A^{*} A}$, and let $\phi(\lambda)=\operatorname{dim}_{\tau}\left(E_{\lambda}\right)=\operatorname{Tr}_{\tau}\left(E_{\lambda}\right)$ denote the corresponding spectral density function. Then define

$$
\operatorname{Det}_{\tau}(A)=\left\{\begin{array}{lc}
\exp \left(\int_{0}^{\infty} \ln (\lambda) d \phi(\lambda)\right) & \text { if the integral is finite } \\
0 & \text { otherwise }
\end{array}\right.
$$

Let $M$ be a finitely generated Hilbertian $\mathcal{A}$-module. Define the determinant line of $M$, denoted by $\operatorname{det} M$, to be the real vector space generated by the symbols $\langle$,$\rangle ,$ one for each admissible scalar product on $M$, with the following relations: $\langle,\rangle_{1},\langle,\rangle_{2}$ are admissible scalar products on $M$. Then

$$
\langle,\rangle_{2}=\operatorname{Det}_{\tau}(A)^{-1 / 2}\langle,\rangle_{1} \quad \text { whenever } \quad\langle v, w\rangle_{2}=\langle A v, w\rangle_{1} \quad \forall v, w \in M,
$$

where $A \in G L(M), A>0$. It has been shown in [CFM] that $\operatorname{det}(M)$ is a real one dimensional vector space.

1.2. $L^{2}$ torsion for covering spaces. We will discuss $L^{2}$ torsion in the special case of normal covering spaces; for the general case of Hilbertian $(\mathcal{A}-\pi)$-bimodules, we refer to $[\mathrm{CFM}],[\mathrm{BFKM}]$.

Let $\Gamma \rightarrow \widehat{M} \rightarrow M$ be a normal covering space of a compact manifold. Let $K$ be a smooth triangulation of $M$, and $\widehat{K}$ be the induced $\Gamma$-invariant triangulation of $\widehat{M}$. Then there is a natural inner product on the space $C^{j}(\widehat{K})$ of finite support co-chains on $\widehat{K}$, where we declare the simplices to be an orthonormal basis. Then $\Gamma$ acts by isometries on $C^{j}(\widehat{K})$, and we consider the Hilbert space completion $C_{(2)}^{j}(\widehat{K})$. The coboundary operator $d^{K}: C^{j}(\widehat{K}) \rightarrow C^{j+1}(\widehat{K})$ induces a bounded operator $d^{\widehat{K}}$ : $C_{(2)}^{j}(\widehat{K}) \rightarrow C_{(2)}^{j+1}(\widehat{K})$. If $d^{\widehat{K} *}$ denotes the $L^{2}$ adjoint of $d^{\widehat{K}}$, then the combinatorial Laplacian

$$
\Delta_{j}^{\widehat{K}}=d^{\widehat{K}} d^{\widehat{K} *}+d^{\widehat{K} *} d^{\widehat{K}}: C_{(2)}^{j}(\widehat{K}) \rightarrow C_{(2)}^{j}(\widehat{K})
$$


is a bounded self adjoint operator, which commutes with the $\Gamma$-action, since $d^{\widehat{K}}$ and $d^{\widehat{K} *}$ do. Let $\Delta_{j}^{\widehat{K}}+$ denote the restriction of $\Delta_{j}^{\widehat{K}}$ onto the orthogonal complement of ker $\Delta_{j}^{\widehat{K}}$. Assume now that $\widehat{M}$ is of determinant class, that is, the Fuglede-Kadison determinant of $\Delta_{j}^{\widehat{K}+}, \operatorname{Det}_{\tau}\left(\Delta_{j}^{\widehat{K}+}\right)$, is defined and is strictly positive for all $j \geq 0$ $[\mathrm{CM}][\mathrm{BFKM}]$. Since $\operatorname{ker} \Delta_{j}^{\widehat{K}} \subset C_{(2)}^{j}(\widehat{K})$, it inherits an admissible scalar product. By the combinatorial analogue of the Hodge theorem, one sees that $\operatorname{ker} \Delta_{j}^{\widehat{K}}$ is isomorphic to the reduced $L^{2}$ cohomology $\bar{H}_{(2)}^{j}(\widehat{K})$. It follows that $\bar{H}_{(2)}^{j}(\widehat{K})$ gets an admissible scalar product, i.e. an element in the $\operatorname{determinant} \operatorname{line} \operatorname{det}\left(\bar{H}_{(2)}^{j}(\widehat{K})\right)$. The alternating tensor product of these elements is an element in $\operatorname{det}\left(\bar{H}_{(2)}^{\bullet}(\widehat{K})\right)$, which we denote by $\hat{\varphi}=\hat{\varphi}(K) \in \operatorname{det}\left(\bar{H}_{(2)}^{\bullet}(\widehat{K})\right)$. The $L^{2}$ Reidemeister-Franz torsion, $\varphi_{\widehat{K}} \in \operatorname{det}\left(\bar{H}_{(2)}^{\bullet}(\widehat{K})\right)$, is defined as in $[\mathrm{CFM}]$ to be

$$
\varphi_{\widehat{K}}=\prod_{j=0}^{n} \operatorname{Det}_{\tau}\left(\Delta_{j}^{\widehat{K}+}\right)^{\frac{(-1)^{j} j}{2}} \hat{\varphi}(K) .
$$

Theorem 1.1. (Topological invariance of $L^{2}$ Reidemeister-Franz torsion [CFM]). Assume that $\widehat{M}$ is of determinant class. Then $\varphi_{\widehat{K}}$ is a topological invariant of $M$ whenever $\operatorname{dim} M$ is odd.

Remark 1.2. When $\bar{H}_{(2)}^{j}(\widehat{K})=0 \quad \forall j \geq 0$, this result was first obtained by [CM]. In fact, $\varphi_{\widehat{K}}$ is an invariant of the simple homotopy type of $K$; see [LuR] and 2.1 below. In the even dimensional case, the $L^{2}$ Reidemeister-Franz torsion is not a topological invariant in general. Also, if $\operatorname{dim} M$ is even and $\bar{H}_{(2)}^{j}(\widehat{K})=0 \quad \forall j \geq 0$, then $\varphi_{\widehat{K}}=1$.

There is also an analytic version of $L^{2}$ Riedemeister-Franz torsion which can be defined in an analogous manner. It is called $L^{2}$ analytic torsion and denoted by $\varphi_{\widehat{M}} \in \operatorname{det}\left(\bar{H}_{(2)}^{\bullet}(\widehat{M})\right)$. We refer to $[\mathrm{CFM}]$ for details of its definition.

Theorem 1.3 (Metric invariance of $L^{2}$ analytic torsion $[\mathrm{CFM}]$ ). Assume that $\widehat{M}$ is of determinant class. Then $\varphi_{\widehat{M}}$ is independent of the choice of Riemannian metric $g$ on $M$ whenever $\operatorname{dim} M$ is odd.

Remark 1.4. When $\bar{H}_{(2)}^{j}(\widehat{M})=0 \forall j \geq 0$, this result was first obtained by $[\mathrm{M}]$ and $[\mathrm{L}]$. In $[\mathrm{CFM}]$, an even more general result is obtained. In the even dimensional case, the $L^{2}$ analytic torsion does in general depend on the choice of Riemannian metric. Also, if $\operatorname{dim} M$ is even and $\bar{H}_{(2)}^{j}(\widehat{M})=0 \forall j \geq 0$, then $\varphi_{\widehat{M}}=1$.

Under the de Rham isomorphism, which identifies $\bar{H}_{(2)}^{j}(\widehat{K})$ and $\bar{H}_{(2)}^{j}(\widehat{M})$ for all $j$ (see $[\mathrm{Do}])$, the results of $[\mathrm{BFKM}]$ are used to prove the following theorem in [CFM].

Theorem 1.5 (Equality of $L^{2}$ torsions $[\mathrm{CFM}]$, [BFKM]). Assume that $\widehat{M}$ is of determinant class. Then via the identification of determinant lines induced by the de Rham isomorphism, the $L^{2}$ analytic torsion and $L^{2}$ Reidemeister-Franz torsion are equal, that is, $\varphi_{\widehat{M}}=\varphi_{\widehat{K}}$, whenever the dimension of $M$ is odd. 


\section{Homotopy INVARIANCE}

Here we establish the homotopy invariance of $L^{2}$ torsion for residually finite covering transformation groups and for amenable covering transformation groups. As mentioned before, in the case when the $L^{2}$ cohomology vanishes and when the covering transformation group is residually finite, this result is due to $[\mathrm{Lu}]$.

We first formulate the problem of the homotopy invariance of $L^{2}$ torsion as follows. Let $f: M \rightarrow N$ be a homotopy equivalence of compact manifolds, with $\widehat{f}$ : $\widehat{M} \rightarrow \widehat{N}$ the induced homotopy equivalence of the normal $\Gamma$-covering spaces $\widehat{M}$ and $\widehat{N}$. Then $\widehat{f}$ induces an isomorphism on the reduced $L^{2}$ cohomology, $\widehat{f}^{*}: \bar{H}_{(2)}^{\bullet}(\widehat{N}) \rightarrow$ $\bar{H}_{(2)}^{\bullet}(\widehat{M})$. By 2.3 in $[\mathrm{CFM}], \widehat{f}^{*}$ induces an isomorphism $\widehat{f}_{*}^{*}$ on determinant lines, $\widehat{f}_{*}^{*}$ : $\operatorname{det} \bar{H}_{(2)}^{\bullet}(\widehat{N}) \rightarrow \operatorname{det} \bar{H}_{(2)}^{\bullet}(\widehat{M})$. Thus a homotopy equivalence of manifolds induces a canonical isomorphism of determinant lines of $L^{2}$ cohomology.

Conjecture 1 (Homotopy invariance of $L^{2}$ torsion). Let $f: M \rightarrow N$ be a homotopy equivalence of compact odd dimensional manifolds. Suppose that $\widehat{M}$ is of determinant class (or equivalently $\widehat{N}$ is of determinant class). Via the identification of determinant lines of $L^{2}$ cohomology of normal $\Gamma$ covering spaces as above, we conjecture that

$$
\phi_{\widehat{M}}=\phi_{\widehat{N}} \in \operatorname{det} \bar{H}_{(2)}^{\bullet}(\widehat{M}) .
$$

This conjecture was first formulated by Lück $[\mathrm{Lu}]$ in the case when the $L^{2}$ cohomology of $M$ is trivial.

Our first result will be a general formula, which generalises the ones in $[\mathrm{LuR}],[\mathrm{Lu}]$ and [Lu1], in the case when the $L^{2}$ cohomology is trivial. Let $A \in G L(n, \mathbb{Z}[\Gamma])$ be an invertible matrix with entries in $\mathbb{Z}[\Gamma]$. Then $A$ defines a bounded invertible operator $R_{A}: \ell^{2}(\Gamma)^{n} \rightarrow \ell^{2}(\Gamma)^{n}$ which commutes with the left action of $\Gamma$ on $\ell^{2}(\Gamma)^{n}$, so it has a positive Fuglede-Kadison determinant $\operatorname{Det}\left(R_{A}\right)$. Also $A$ defines an element in the Whitehead group of $\Gamma$, and it can be shown that the Fuglede-Kadison determinant Det $_{\tau}$ induces a homomorphism

$$
\Phi_{\Gamma}: W h(\Gamma) \rightarrow \mathbb{R}^{+}
$$

which was defined in $[\mathrm{LuR}]$ and $[\mathrm{Lu}]$. Now a homotopy equivalence $f: M \rightarrow N$ defines an acyclic complex $C^{\bullet}\left(M_{f}\right)$ of cochains over the group ring of $\Gamma$, where we now choose cell decomposition for $M$ and $N$, and $f$ to be a cellular homotopy equivalence and $M_{f}$ the cellular mapping cone. Then as in [Mi], this acyclic complex defines the Whitehead torsion $T(f) \in W h(\Gamma)$ of the homotopy equivalence $f$. Note that via the identification above, one has $\phi_{\widehat{M}} \otimes \phi_{\widehat{N}}^{-1} \in \mathbb{R}$. Then we have the following:

Proposition 2.1. Let $f: M \rightarrow N$ be a homotopy equivalence of compact odd dimensional manifolds. Suppose that $\widehat{M}$ is of determinant class. Via the identification of determinant lines of $L^{2}$ cohomology of normal $\Gamma$-covering spaces as above, one has

$$
\phi_{\widehat{M}} \otimes \phi_{\widehat{N}}^{-1}=\Phi_{\Gamma}(T(f)) \in \mathbb{R}^{+} .
$$

To prove this proposition, we recall some definitions and facts from $[\mathrm{CFM}]$ about abstract determinant class complexes. We begin with

Definition 2.2. Consider a finitely generated Hilbertian module $M$ over $\mathcal{A}$. A scalar product $\langle$,$\rangle on M$ will be called $D$-admissible if it can be represented in the 
form

$$
\langle v, w\rangle=\langle A(v), w\rangle_{1} \quad \text { for } \quad v, w \in M,
$$

where $\langle,\rangle_{1}$ is an admissible scalar product on $M$ and $A \in B(M)$ is an injective (possibly not invertible) homomorphism $A: M \rightarrow M$, which is positive and selfadjoint with respect to $\langle,\rangle_{1}$, such that $\operatorname{Det}_{\tau}(A)>0$.

By a morphism of Hilbertian modules, we always mean one which preserves the modules structure over the von Neumann algebra. An injective homomorphism of Hilbertian modules $f: M \rightarrow N$ with dense image will be called a $D$-isomorphism if for some, and therefore every, admissible scalar product $\langle,\rangle_{N}$ on $N$, the induced scalar product $\langle,\rangle_{M}$ on $M$ given by $\langle v, m\rangle_{M}=\langle f(v), f(w)\rangle_{N}$, is $D$-admissible. Note that any $D$-isomorphism of Hilbertian modules $f: M \rightarrow N$ induces an isomorphism of determinant lines $f_{*}: \operatorname{det}(M) \rightarrow \operatorname{det}(N)$, defined above; cf. [CFM].

A sequence of Hilbertian modules and morphisms $0 \rightarrow M^{\prime} \stackrel{\alpha}{\rightarrow} M \stackrel{\beta}{\rightarrow} M^{\prime \prime} \rightarrow 0$ is called $D$-exact is $\alpha$ is a monomorphism, $\operatorname{im} \alpha=\operatorname{ker} \beta$ and the map $M / \operatorname{ker} \beta \rightarrow M^{\prime \prime}$ induced by $\beta$ is a $D$-isomorphism.

Let

$$
0 \rightarrow C^{0} \stackrel{d}{\rightarrow} C^{1} \stackrel{d}{\rightarrow} \cdots \stackrel{d}{\rightarrow} C^{N} \rightarrow 0
$$

be a cochain complex of finite length formed by finitely generated Hilbertian $\mathcal{A}$ modules and bounded linear maps commuting with the action of $\mathcal{A}$. Here $\mathcal{A}$ is a finite von Neumann algebra, such as the group von Neumann algebra of $\Gamma$. Let $Z^{i}$ denote the submodule of cocycles and $B^{i}$ the submodule of coboundaries. Then the cochain complex $C^{\bullet}$ is said to be of determinant class if the sequence

$$
0 \rightarrow Z^{i} \stackrel{\alpha_{i}}{\rightarrow} C^{i} \stackrel{\beta_{i}}{\rightarrow} \bar{B}^{i} \rightarrow 0
$$

is $D$-exact, that is, $\operatorname{im}\left(\alpha_{i}\right)=\operatorname{ker}\left(\beta_{i}\right)$ and the map $C^{i} / \operatorname{ker}\left(\beta_{i}\right) \rightarrow \bar{B}^{i}$ induced by $\beta_{i}$ is a $D$-isomorphism. This agrees with the notion of determinant class discussed in section 1.2 (cf. [CFM]).

Definition 2.3. Let $C^{\bullet}$ be a cochain complex which is of determinant class. Then by Proposition 3.10 of $[\mathrm{CFM}]$ there is a canonical isomorphism between the determinant lines of the graded Hilbertian modules,

$$
\phi_{C}: \operatorname{det}(C) \rightarrow \operatorname{det}(\bar{H}(C)),
$$

where $\bar{H}(C)$ denotes the reduced $L^{2}$ cohomology. This gives a definition of the $L^{2}$ torsion of an abstract cochain complex $C^{\bullet}$ which is of determinant class,

$$
\phi_{C} \in \operatorname{det}(C)^{-1} \otimes \operatorname{det}(\bar{H}(C)) .
$$

Let $K$ be a smooth triangulation on $M$, and $\widehat{K}$ be the induced $\Gamma$-invariant triangulation on $\widehat{M}$. It can be shown that the $L^{2}$ torsion of the $L^{2}$ cochain complex $C_{(2)}^{\bullet}(\widehat{K})$ of $\widehat{K}$ is equal to the $L^{2}$ Reidemeister-Franz torsion of $\widehat{K}$ (see Proposition 3.11 in $[\mathrm{CFM}])$.

Proposition 2.4. Let $f: C \rightarrow C^{\prime}$ be a cochain homotopy equivalence of determinant class complexes. Then $C_{f}$ is of determinant class and

$$
\phi_{C^{\prime}}^{-1} \otimes \phi_{C}=\phi_{C_{f}} \in \operatorname{det}\left(C_{f}\right)^{-1} \text {, }
$$

where $C_{f}$ denotes the mapping cone complex. 
Proof. There is a short exact sequence

$$
0 \rightarrow C^{\prime-1} \rightarrow C_{f} \rightarrow C \rightarrow 0,
$$

where $C^{\prime-1}$ denotes the complex $C^{\prime}$ shifted in degree by -1 . Now $C_{f}$ is acyclic and therefore is of determinant class. By Proposition 3.5 of [CFM], one has canonical isomorphisms

$$
\operatorname{det}\left(C^{\prime-1}\right) \otimes \operatorname{det}(C) \rightarrow \operatorname{det}\left(C_{f}\right) \quad \text { and } \quad \operatorname{det}\left(\bar{H}\left(C^{\prime-1}\right)\right) \otimes \operatorname{det}(\bar{H}(C)) \rightarrow \mathbb{C} .
$$

Therefore we get the canonical isomorphism

$$
\operatorname{det}\left(C^{\prime}\right) \otimes \operatorname{det}\left(\bar{H}\left(C^{\prime}\right)\right)^{-1} \otimes \operatorname{det}(C)^{-1} \otimes \operatorname{det}(\bar{H}(C)) \rightarrow \operatorname{det}\left(C_{f}\right)^{-1},
$$

which yields the identity $\phi_{C^{\prime}}^{-1} \otimes \phi_{C}=\phi_{C_{f}} \in \operatorname{det}\left(C_{f}\right)^{-1}$.

Proof of Proposition 2.1. By Proposition 2.4, it suffices to determine the quantity $\phi_{C_{f}} \in \operatorname{det}\left(C_{f}\right)^{-1}$ in our particular case. First, since the complexes and the exact sequences are based, we see that $\phi_{C_{f}} \in \mathbb{R}$. Next, $C_{f}$ defines the Whitehead torsion $T(f) \in W h(\Gamma)$, and it is clear that the $L^{2}$ torsion $\phi_{C_{f}}$ is just the Fuglede-Kadison determinant of the Whitehead torsion $T(f), \Phi_{\Gamma}(T(f))$.

Proposition 2.5. Suppose that $\Gamma$ is a finitely presented amenable group. Then the homomorphism

$$
\Phi_{\Gamma}: W h(\Gamma) \rightarrow \mathbb{R}^{+}
$$

is trivial.

Proof. Let $a \in W h(\Gamma)$. Then by [Mi], it is represented as the Whitehead torsion of a homotopy equivalence $f: L \rightarrow K$ of finite CW complexes, which we can assume without loss of generality is an inclusion, i.e. $a=T(f)$. Let $\widehat{K}$ and $\widehat{L}$ denote the corresponding $\Gamma$ normal covering complexes. The relative cochain complex $C(\widehat{K}, \widehat{L})$ is acyclic, and so is its $L^{2}$ completion $C_{(2)}(\widehat{K}, \widehat{L})$. In particular, the combinatorial Laplacian $\Delta_{j}^{\widehat{K}, \widehat{L}}$ is invertible, and we see that

$$
\Phi_{\Gamma}(T(f))=\prod_{j=0}^{n} \operatorname{Det}_{\tau}\left(\Delta_{j}^{\widehat{K}, \widehat{L}}\right)^{\frac{(-1)^{j} j}{2}}>0 .
$$

Let $\mathcal{F}_{K}$ denote a fundamental domain for the action of the group $\Gamma$ on $\widehat{K}$, and let $\mathcal{F}_{L}=\mathcal{F}_{K} \cap \widehat{L}$, which is a fundamental domain for the action of $\Gamma$ on $\widehat{L}$.

Since $\Gamma$ is amenable, by the $\mathrm{F} \varnothing$ lner criterion for amenability one gets a regular exhaustion, that is, a sequence $\left\{X_{m}\right\}_{m=1}^{\infty}$ of finite subsets of $\Gamma$ such that

$$
\Gamma=\bigcup_{m=1}^{\infty} X_{m} \quad \text { and } \quad \lim _{m \rightarrow \infty} \frac{\sharp \partial_{\delta} X_{m}}{\sharp X_{m}}=0,
$$

where $\partial_{\delta} X_{m}=\left\{\gamma \in \Gamma: d\left(\gamma, X_{m}\right)<\delta\right.$ and $\left.d\left(\gamma, \Gamma-X_{m}\right)<\delta\right\}$ is a $\delta$-neighbourhood of the "boundary" of $X_{m}, d(\cdot, \cdot)$ denotes the word metric on $\Gamma$ and $\sharp X_{m}$ denotes the number of elements in $X_{m}$.

One then gets regular exhaustions $\left\{L_{m}\right\}_{m=1}^{\infty}$ of $\widehat{L}$ and $\left\{K_{m}\right\}_{m=1}^{\infty}$ of $\widehat{K}$, that is, sequences of finite subcomplexes of $\widehat{L}$ and $\widehat{K}$ respectively such that

(1) $L_{m}=\bigcup_{g \in X_{m}} g . \mathcal{F}_{L}$ and $K_{m}=\bigcup_{g \in X_{m}} g . \mathcal{F}_{K}$;

(2) $\widehat{L}=\bigcup_{m=1}^{\infty} L_{m}$ and $\widehat{K}=\bigcup_{m=1}^{\infty} K_{m}$; 
(3) Let $\dot{N}_{m, \delta}$ denote the number of elements $g \in \Gamma$ which have distance (with respect to the word metric in $\Gamma$ ) less than or equal to $\delta$ from any element $g^{\prime} \in \Gamma$ such that the intersection of $g^{\prime} . \mathcal{F}_{L}$ with the topological boundary $\partial L_{m}$ of $L_{m}$ is not empty, that is, $\dot{N}_{m, \delta}=\sharp \partial_{\delta} X_{m}$. Let $N_{m}=\sharp X_{m}$, which also equals the number of translates of $\mathcal{F}_{L}$ which are contained in $L_{m}$. Then by the amenability assumption, one has for every $\delta>0$,

$$
\lim _{m \rightarrow \infty} \frac{\dot{N}_{m, \delta}}{N_{m}}=0 .
$$

Note that $\dot{N}_{m, \delta}$ also equals the number of elements $g \in \Gamma$ which have distance (with respect to the word metric in $\Gamma$ ) less than or equal to $\delta$ from any element $g^{\prime} \in \Gamma$ such that the intersection of $g^{\prime} . \mathcal{F}_{K}$ with the topological boundary $\partial K_{m}$ of $K_{m}$ is not empty, and $N_{m}$ also equals the number of translates of $\mathcal{F}_{K}$ which are contained in $K_{m}$.

We can then define the relative cochain complexes $C\left(K_{m}, L_{m}\right)$ (note that $L_{m}=$ $\left.K_{m} \cap \widehat{L}\right)$. Let $\Delta_{j}^{(m)}$ denote the combinatorial Laplacian acting on $C^{j}\left(K_{m}, L_{m}\right)$. Let $\Delta_{j}^{(m)+}$ denote the combinatorial Laplacian acting on the orthogonal complement of its null-space. Then by the results of section 4 in [DM2], which are easily generalised to the relative situation which is being considered here, one has

$$
\operatorname{Det}_{\tau}\left(\Delta_{j}^{\widehat{K}, \widehat{L}}\right) \geq \lim \sup _{m \rightarrow \infty} \operatorname{det}_{\mathbb{C}}\left(\Delta_{j}^{(m)+}\right)^{\frac{1}{N_{m}}}=1
$$

since $\Delta_{j}^{(m)+}$ is an invertible matrix with integer entries. To prove equality, we notice that if $E_{m}(\lambda)$ denotes the spectral density function of $\Delta_{j}^{(m)}$, then

$$
F_{m}(\lambda)=F_{m}(0) \text { for all } \lambda<K^{-2}, m \geq 0,
$$

where $F_{m}(\lambda)=\frac{E_{m}(\lambda)}{N_{m}}$ and $K$ is a constant as in Lemma 2.2 in [DM2] such that

$$
K^{2} \geq \max \left\{\left\|\Delta_{j}^{\widehat{K}, \widehat{L}}\right\|,\left\|\Delta_{j}^{(m)+}\right\|,\left\|\Delta_{j}^{(m)+{ }^{-1}}\right\|,\left\|\Delta_{j}^{\widehat{K}, \widehat{L}^{-1}}\right\|\right\}
$$

for all $m \geq 0$. Actually in Lemma 2.2 in [DM2], one obtains a constant $K_{1}$ such that

$$
K_{1}^{2} \geq \max \left\{\left\|\Delta_{j}^{\widehat{K}, \widehat{L}}\right\|,\left\|\Delta_{j}^{(m)+}\right\|\right\}
$$

for all $m \geq 0$. Then an identical proof as in Lemma 2.2 in [DM2] gives the existence of a constant $K_{2}$ such that

$$
K_{2}^{2} \geq \max \left\{\left\|\Delta_{j}^{(m)+{ }^{-1}}\right\|,\left\|\Delta_{j}^{\widehat{K}, \widehat{L}^{-1}}\right\|\right\} .
$$

Finally, choose $K$ to be the greater of $K_{1}, K_{2}$.

The estimate $(2.1)$ says that $\Delta_{j}^{(m)}$ has a spectral gap at zero which is bounded away from zero, independent of $m \geq 0$. Let $\bar{F}(\lambda)=\lim \sup _{m \rightarrow \infty} F_{m}(\lambda)$, where we assume without loss of generality that $\bar{F}(\lambda)$ is right continuous. By Theorem 2.1 in [DM2], one has $\bar{F}(\lambda)=F(\lambda)$, where $F(\lambda)$ denotes the spectral density function of $\Delta_{j}^{\widehat{K}, \widehat{L}}$. Therefore

$$
\begin{gathered}
0 \leq \bar{F}(\lambda)-\bar{F}(0)=\lim \sup _{m \rightarrow \infty} F_{m}(\lambda)-\lim _{m \rightarrow \infty} F_{m}(0) \\
\leq \sup \left\{\left|F_{m}(\lambda)-F_{m}(0)\right|: m \geq 0\right\}=0
\end{gathered}
$$


if $\lambda<K^{-2}$, by (1). Therefore one has

$$
F(\lambda)=\bar{F}(\lambda)=\bar{F}(0) \quad \text { for } \quad \lambda<K^{-2} .
$$

The estimate (2.2) says that $\Delta_{j}^{\widehat{K}, \widehat{L}}$ also has a spectral gap of size at least $K^{-2}$, at zero. Also observe that

$$
\begin{aligned}
& \int_{0}^{K^{2}} \sup \left\{F_{m}(\lambda)-F_{m}(0): m \geq 0\right\} d \lambda \\
& \quad=\int_{K^{-2}}^{K^{2}} \sup \left\{F_{m}(\lambda)-F_{m}(0): m \geq 0\right\} d \lambda<\infty .
\end{aligned}
$$

By the dominated convergence theorem, one sees as in Lemma 3.3.1 in [Lu] that

$$
\int_{0+}^{K^{2}} \frac{F(\lambda)-F(0)}{\lambda} d \lambda=\lim _{m \rightarrow \infty} \int_{0+}^{K^{2}} \frac{F_{m}(\lambda)-F_{m}(0)}{\lambda} d \lambda .
$$

Since, by definition,

$$
\begin{aligned}
\frac{1}{N_{m}} \log \operatorname{det}_{\mathbb{C}}\left(\Delta_{j}^{(m)+}\right) & =\int_{0+}^{K^{2}} \log \lambda d F_{m}(\lambda) \\
& =\log K^{2}\left(F_{m}\left(K^{2}\right)-F_{m}(0)\right)-\int_{0+}^{K^{2}} \frac{F_{m}(\lambda)-F_{m}(0)}{\lambda} d \lambda, \\
\log \operatorname{Det}_{\tau}\left(\Delta_{j}^{\widehat{K}, \widehat{L}}\right) & =\int_{0+}^{K^{2}} \log \lambda d F(\lambda) \\
& =\log K^{2}\left(F\left(K^{2}\right)-F(0)\right)-\int_{0+}^{K^{2}} \frac{F(\lambda)-F(0)}{\lambda} d \lambda,
\end{aligned}
$$

it follows by the equality (2.3) above and Theorem 2.1 in [DM2] that

$$
\operatorname{Det}_{\tau}\left(\Delta_{j}^{\widehat{K}, \widehat{L}}\right)=\lim \sup _{m \rightarrow \infty} \operatorname{det}_{\mathbb{C}}\left(\Delta_{j}^{(m)+}\right)^{\frac{1}{N_{m}}}=1 .
$$

This completes the proof of the proposition.

Combining Propositions 2.1 and 2.5 in this section, and using Theorem 0.5, part a) in $[\mathrm{Lu}]$, which states that

$$
\Phi_{\Gamma}: W h(\Gamma) \rightarrow \mathbb{R}^{+}
$$

is trivial whenever $\Gamma$ is residually finite, and using Theorem 1.5 of the previous section, we obtain the following theorem, which gives significant evidence for the conjecture stated at the begining of the section.

Theorem 2.6. Let $f: M \rightarrow N$ be a homotopy equivalence of compact, odd dimensional manifolds. Via the identification of determinant lines of $L^{2}$ cohomology of normal $\Gamma$ covering spaces as in the beginning of the section, and whenever $\Gamma$ is either residually finite or $\Gamma$ is amenable, one has

$$
\phi_{\widehat{M}}=\phi_{\widehat{N}} \in \operatorname{det} \bar{H}_{(2)}^{\bullet}(\widehat{M}) \text {. }
$$




\section{Applications}

In this section, we briefly give some applications of Theorem 2.6. We begin with

Proposition 3.1 ( $L^{2}$ torsion for discrete groups). Let $\Gamma$ be either a residually finite group or an amenable group, whose classifying space $B \Gamma$ is a finite $C W$ complex of odd dimension. Then the $L^{2}$ torsion of $\Gamma$, defined as

$$
\phi_{\Gamma}=\phi_{E \Gamma} \in \operatorname{det}\left(\bar{H}_{(2)}^{\bullet}(\Gamma)\right) \text {, }
$$

is well defined and depends only on the isomorphism class of $\Gamma$.

Proof. By hypothesis and results of [Lu], [BFK] and [DM2], EГ is of determinant class. Now $B \Gamma$ is only well defined up to homotopy, and since the determinant class condition depends only on the homotopy type of $B \Gamma$ (cf. [BFKM]), and by Theorem 2.6 , the $L^{2}$ torsion is a homotopy invariant. Therefore $\phi_{E \Gamma}$ is well defined.

It is therefore natural to conjecture the following,

Conjecture 2. Let $\Gamma$ be a discrete group whose classifying space $B \Gamma$ is a finite $C W$ complex of odd dimension. Then one can define the $L^{2}$ torsion of $\Gamma$ as

$$
\phi_{\Gamma}=\phi_{E \Gamma} \in \operatorname{det}\left(\bar{H}_{(2)}^{\bullet}(\Gamma)\right) .
$$

Our next application is a new proof of the homotopy invariance of the hyperbolic volume of closed 3-dimensional hyperbolic manifolds. Although it uses Theorem 2.6 , it uses only the part that was proved by Lück [Lu].

Proposition 3.2 (Homotopy invariance of hyperbolic volume). Let $M$ and $N$ be homotopy equivalent closed 3-dimensional hyperbolic manifolds. Then their hyperbolic volumes are equal.

Proof. By the calculations in $[\mathrm{M}]$ and $[\mathrm{L}]$, one knows that the $L^{2}$ torsions of $\widetilde{M}$ and $\widetilde{N}$ are proportional to the hyperbolic volumes of $M$ and $N$ respectively, i.e.

$$
-6 \pi \log \phi_{\widetilde{M}}=\operatorname{vol}(M) \quad \text { and } \quad-6 \pi \log \phi_{\widetilde{N}}=\operatorname{vol}(N) .
$$

Now applying Theorem 1.5 and Theorem 2.6, we see that $\operatorname{vol}(M)=-6 \pi \log \phi_{\widetilde{M}}=$ $-6 \pi \log \phi_{\widetilde{N}}=\operatorname{vol}(N)$.

\section{REFERENCES}

[ASS] H. Araki, M-S.B. Smith and L. Smith, On the homotopical significance of the type of von Neumann algebra factors, Commun. Math. Phys. 22 (1971), 71-88. MR 44:5783

[BFKM] D. Burghelea, L. Friedlander, T. Kappeler and P. McDonald, Analytic and Reidemeister torsion for representations in finite type Hilbert modules, Geom. Funct. Anal. 6 (1996), 751-858. MR 97i:58177

[BFK] D. Burghelea, L. Friedlander and T. Kappeler, Torsion for manifolds with boundary and glueing formulae, preprint 1996.

[CM] A. Carey and V. Mathai, $L^{2}$ Torsion Invariants, Journal of Functional Analysis 110 (1992), 377-409. MR 94a:58211

[CFM] A. Carey, M. Farber and V. Mathai, Determinant Lines, Von Neumann Algebras and $L^{2}$ torsion, J. Reine Angew. Math. 484 (1997), 153-181. CMP 97:09

[Di] J. Dixmier, Von Neumann algebras, North-Holland, Amsterdam (1981). MR 83a:46004

[Do] J. Dodziuk, De Rham-Hodge theory for $L^{2}$ cohomology of infinite coverings, Topology 16 (1977), 157-165. MR 56:3898

[DM] J. Dodziuk and V. Mathai, Approximating $L^{2}$ invariants of amenable covering spaces: A heat kernel approach, Lipa's Legacy (Proc. Bers Colloq., 1995; J. Dodziuk and L. Keen, editors), Contemp. Math., vol. 211, Amer. Math. Soc., Providence, RI, 1997, pp. $151-167$. 
[DM2] J. Dodziuk and V. Mathai, Approximating $L^{2}$ invariants for amenable covering spaces: A combinatorial approach, to appear in Journal of Functional Analysis.

[FJ] F.T. Farrell and L.E. Jones, Isomorphism conjectures in algebraic K-theory, JAMS 6 (1993), 249-298. MR 93h:57032

[FK] B. Fuglede and R.V. Kadison, Determinant theory in finite factors, Annals of Math. 55 (1952), 520-530. MR 14:660a

[L] J. Lott, Heat kernels on covering spaces and topological invariants, J. Diff. Geom. 35 (1992), 471-510. MR 93b:58140

[Lu] W. Lück, Approximating $L^{2}$ invariants by their finite dimensional analogues, Geom. and Func. Analysis 4 (1994), 455-481. MR 95g:58234

[Lu1] W. Lück, $L^{2}$-Torsion and 3-manifolds, Low-Dimensional Topology (Knoxville, TN, 1992; K. Johannson, editor), Conf. Proc. and Lecture Notes Geom. Topology, vol. III, Internat. Press, Cambridge, MA, 1994, pp. 75-107. MR 96g:57019

[LuR] W. Lück and M. Rothenberg, Reidemeister torsion and the K-theory of von Neumann algebras, K-Theory 5 (1991), 213-264. MR 93g:57025

[M] V. Mathai, $L^{2}$ analytic torsion, J. Func. Anal. 107 (1992), 369-386. MR 93g:58146

[Mi] J. Milnor, Whitehead torsion, Bull. Amer. Math. Soc., 72, (1966), 358-426. MR 33:4922

[RS] D. Ray and I. M. Singer, R-torsion and the Laplacian on Riemannian manifolds, Advances in Math. 7 (1971), 145-210. MR 45:4447

Department of Mathematics, University of Adelaide, Adelaide 5005, Australia

E-mail address: vmathai@maths.adelaide.edu.au

Department of Mathematics, University of Chicago, Chicago, Illinois 60637

E-mail address: mel@math.uchicago.edu 